\title{
Treatment of Neuroendocrine Tumors (NETs) Using Somatostatin Analogs: Current View, Clinical Achievements and Future Perspectives
}

\author{
Hendris Wongso* \\ Labelled Compound and Radiometry Division, Center for Applied Nuclear Science and Technology, National Nuclear \\ Energy Agency (BATAN), Bandung, Indonesia
}

\begin{abstract}
Neuroendocrine tumors (NETs), previously known as carcinoid tumors, are a heterogeneous group of neoplasms which originate from cells of the endocrine or nervous system with an overall incidence of 1-5 cases per 100,000 individuals per year. Recent studies showed that their incidence has dramatically increased worldwide over the last few years. The majority of NETs overexpress the somatostatin receptors (SSTRs) in tumor cells which are further being used as the important targets for therapy purposes using somatostatin analogs (SSAs) such as octreotide and lanreotide. Like native somatostatin, SSAs bind to SSTRs and induce a range of cellular effects, including antitumor activity. Antitumor activity of SSAs and their high binding properties for the SSTRs have become valuable tools for developing advanced treatment of NETs. Consequently, SSAs have been used widely in routine clinical practice, especially for treatment of various types of NETs. Despite treatment using SSAs have made a positive contribution, recent development suggests that the used of SSAs in combination with chemotherapy or peptide receptor radionuclide therapy (PRRT) can improve clinical outcome in patients with NETs. This review provides an overview of the recent trend in the treatment of NETs using SSAs, their clinical achievements in the last few years and their potential applications in the future.
\end{abstract}

Keywords: neuroendocrine tumors, treatment, somatostatin analogs, chemotherapy, PRRT

\section{INTRODUCTION}

Neuroendocrine tumors (NETs), previously known as carcinoid tumors are a heterogeneous group of neoplasms which originate from cells of the endocrine or nervous system and may derive from the embryonic neural crest cells of several organs (Kaltsas, et al., 2004; Gould, et al., 2013; Al-Chalabi, et al., 2018). Most NETs are located in the gastrointestinal tract and the lung and occasion- ally occur in the pancreas, head and neck region, prostate, ovary, testis and liver (Gabriel, et al., 2007; Modlin, et al., 2003). Interestingly, NETs can also be found in the breast tissue, although the incidence

Submitted: May 20, 2019

Revised: June 23, 2019

Accepted: June 24, 2019

*Corresponding author: hendriswongso@batan.go.id 
is rare, around $0.27-0.50 \%$ (Adams, et al., 2014). Despite pathophysiological mechanism underlying NETs is still largely unknown, study at the genomic level has suggested the important role of transcriptome, micro-RNome and exome in the regulation of secretory and proliferative mechanism of NETs (Kidd, et al., 2015). In addition, several hypotheses including dysregulation of the phosphatidylinositol-3-kinase (PI3K)/AKT and the mammalian target of rapamycin (mTOR) signaling have been linked with the pathogenesis of these tumors (Boora, et al., 2015).

NETs are characterized by their ability to overexpress the neurosecretory granules that contain numerous bioactive compounds such as peptides, hormones, kinins, vasoactive amines, and endorphins which cause characteristic hormonal syndromes leading to several clinical signs and symptoms (Gould, et al., 2013; Yao, et al., 2008). A variety of clinical symptoms in NETs patients may include flushing, diarrhea, bronchospasm, hepatomegaly, sweating, hemoptysis, wheeze, hypotension and anxiety (Bodei, et al., 2014; Jayasena, et al., 2013). Additionally, local symptoms induced by tumor aggregates (e.g., pain, bleeding, obstruction, and perforation) are frequently observed in patients with advanced NETs (Frilling, et al., 2014). Importantly, the majority of NETs overexpress somatostatin receptors (SSTRs), mainly types 2 and 5 on their cell surfaces which are further being used as the important targets for therapy of patients with NETs and neuroendocrine neoplasias (NENs) (Kayani, et al., 2008; García-Talavera, et al., 2014; Fani, et al., 2017).

It was previously believed that NETs are relatively slow growing and uncommon in humans with an overall incidence of $1-5$ cases per 100,000 per year (Amr, et al., 2015; van der Zwan, et al., 2013). However, a large number of reports showed that their incidence has dramatically increased worldwide over the last few years. According to Surveillance Epidemiology and End Results (SEER) database, in 1973 the incidence of NETs was 1.09 per 100,000 . This incidence increased 5- fold in 2004 to 5.25 per 100,000 (Yao, et al., 2008). Furthermore, the project of surveillance of rare cancer (RARECARE) also found that the overall incidence rate of NETs in Europe was 25 per 1,000,000 during 1978-2002 (van der Zwan, et al., 2013).

In the United Kingdom during 1971-2006, the incidence of NETs rose approximately 2.9- to 3.8-fold (Frilling, et al., 2014). In particular, since the 1970s, the incidence of pancreatic endocrine tumor (pNET) also increased annually from 0.32 to 0.43 per 100,000 (Dilz, et al., 2015). Korse et al. reported that the incidence of grade 1 NET (G1NET) in the Netherlands has progressively increased from 2 per 100,000 in 1990 to 3 per 100,000 in 2010 and the large increase experienced by grade 2 NET (G2NET) from 0.01 per 100,000 in 1990 to 0.2 per 100,000 in 2010 (Korse, et al., 2013). Another report showed that in Denmark, the incidence of NETs increased by $2-$ fold in men from 0.24 to 0.53 per 100,000 per year (Skuladottir, et al., 2002). In Australia, the annual incidence of NETs increased approximately 2-fold from 1.7 (1980-1989) to 3.3 (2000-2006) (Luke, et al., 2010).

An important progress in the field of NETs management was the recent development of several therapeutic strategies that exploit the role of somatostatin and its analogs, which seemed to become an attractive option in routine clinical practice (Rai, et al., 2015). Although the application of somatostatin analogs (SSAs) is a well established treatment for various types of NETs, the combination of SSAs with other therapies has not been extensively studied. Thus, current development in preclinical and clinical studies of SSAs for treatment of NETs and their combination with chemotherapy and peptide receptor radionuclide therapy (PRRT) as well as the potential future of therapeutic applications are discussed here.

\section{Somatostatin and Somatostatin Receptors (SSTRs)}

Somatostatin, also known as somatotropin release-inhibiting factor is a naturally occurring peptide hormone that controls the endocrine and 
exocrine secretion and inhibits proliferation of normal and tumor cells (Susini, et al., 2006). The role of somatostatin in NETs is mediated through its interaction with one of five SSTRs (Baldelli, et al., 2014). Therefore, the correlation of NETs with the SSTRs has raised significant interest among clinicians and scientists worldwide, in particular for developing a more suitable treatment for the patients with SSTR-expressing NETs, informed by the wide range of pharmacological studies reported in recent years (Chalabi, et al., 2014).

Based on the correlation study of mRNA and maximum standardized uptake value $\left(\mathrm{SUV}_{\max }\right)$, SSTR-1 was the highest level of receptor in the normal human tissue. Similarly, SSTR-2 was nearly as high as SSTR-1. Other somatostatin receptors showed lower levels and SSTRs -4 is the lowest abundance receptor (Boy, et al., 2011). Several studies have revealed that SSTRs abundance in NETs were significantly higher than normal tissues. In vivo biodistribution study using SSA agonist radiotracer ${ }^{99 \mathrm{~m}} \mathrm{Tc}$-[ethylenediamine- $N, N^{\prime}$-diacetic acid/tricine/6-hydrazinopyridine-3-carboxylic acid $\left(\mathrm{HYNIC}^{0}\right)$ ] 1-Nal ${ }^{3}, \mathrm{Thr}^{8}-$ octreotide $\left({ }^{99 \mathrm{~m}} \mathrm{Tc}-[\mathrm{EDDA} /\right.$ tricine/HYNIC]-NATE) demonstrated that SSTRs accumulation in rat bearing AR4-2J tumor was higher than blood, adrenals, spleen, stomach, intes- tine, liver, lung, heart and bone after 1 and 4 hours injection, respectively (Gandomkar, et al., 2007). Moreover, it was reported that the biodistribution and accumulation of SSA antagonist radiotracer ${ }^{111} \mathrm{In}-1,4,7,10$-tetraazacyclododecane-1,4,7,10tetraacetic acid (DOTA)-sst2-antagonist (ANT) ( ${ }^{111}$ In-DOTA-sst2-ANT) was higher in NET than other organs for 4 hours after injection (Wang, et al., 2012).

In many cases, NETs are express varying concentrations of SSTRs. Thus, the abundance of SSTR is depending on the origin, stage, and the type of tumor. SSTR-1 can be found in high concentration in prostate cancer tissues, although other SSTRs are present. Generally, all SSTR subtypes are expressed in NETs, however, SSTR-2 was found in higher level compared to others subtypes (Cakir, et al., 2010). The significant expression of SSTR-2 and SSTR-5 in humans was reported in 108 endocrine pituitary tumors study. This study revealed that $93 \%$ of growth hormone tumors expressed SSTR-2 and 83\% expressed SSTR-5 (Chinezu, et al., 2014). Other tumors such as thyroid, melanoma and gastrointestinal carcinoid expressed all five receptors although in varying level. In specific tumors such as hepatocellular carcinoma, the SSTR-5 expression was dominant (Blaker, et al., 2004).

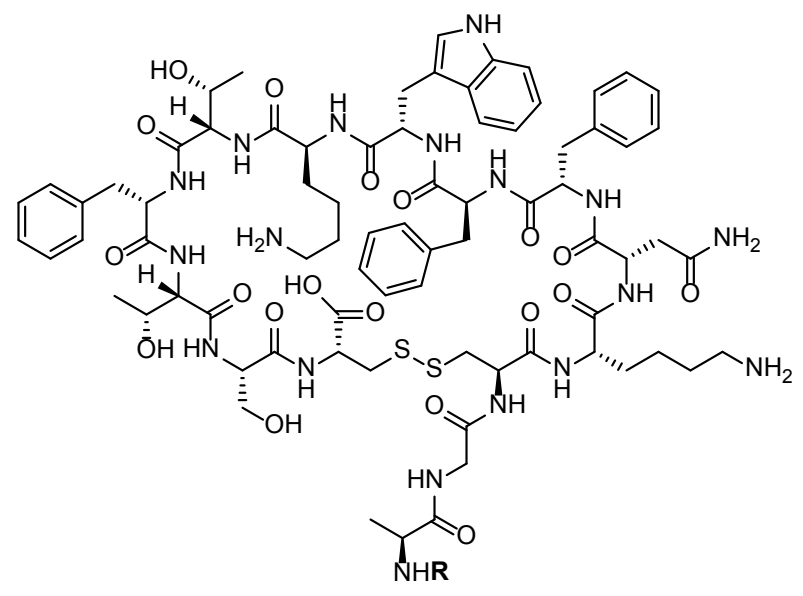

Figure 1. Chemical structures of the native somatostatin. $R=H$ (somatostatin-14); $R=H$-serine-alanine-asparagine-serine-asparagine-proline-alanine-methionine-alanine-proline-arginine-glutamic acid-arginine-lysine (somatostatin-28) (Weckbecker, et.al., 2003) 


\section{NETs Treatment using Somatostatin Analogs (SSAs)}

Initially, surgery is the established treatment and has been used for a long time to remove NETs and prolong survival rate in patients (Kaltsas, et al., 2004; Ha, et al., 2012; Walter, et al., 2012). This treatment has also been used in patients with liver metastasis which arises from carcinoid tumors (Pathak, et al., 2013; Nave, et al., 2001). A study showed that surgery for cytoreduction of NET metastasis has been associated with the duration of survival, for instance Mayo and colleagues reported that a 5-year survival and 10-year survival of $74 \%$ and $51 \%$ respectively, were achieved by the patients after liver-directed surgery for metastatic NETs (Mayo, et al., 2010). However, the surgery outcome is directly depending on the stage of tumors (tumor size) (Ha, et al., 2012), tumor location, and involvement of surrounding tissues (Oberg, 2012).

Another treatment to destroy the tumor aggregates of NETs is chemotherapy. This approach has traditionally been used in a variety of NETs. The use of combination chemotherapeutic agents, such as 5-fluorouracil and streptozotocin generated an overall disease control (ODC) rate of $83.3 \%$ in 96 patients with pNET (Dilz, et al., 2015). Moreover, single agent for NETs therapy such as adriamycin, 5-fluorouracil, actinomycin-D and dacarbazine demonstrated an objective response (OR) of $21,26,6$, and $13 \%$, respectively (Moertel, 1983). In NETs, the efficacy of chemotherapy is always associated with the types of NETs, for example, pNETs have a high sensitivity to chemotherapy, whereas gastrointestinal NETs (GINETs) are often resistant to chemotherapy treatment (Walter, et al., 2012). Moreover, due to the toxicity, some chemotherapy agents may be not suitable for some patients and therefore limit their use in clinical setting (Peixoto, et al., 2014).

NETs management has evolved over the last few decades due to the increasing understanding of their tumor biology and molecular regulation.
Some studies have been showed that somatostatin has a broad range of biological functions such as antiproliferative regulation (Owonikoko, et al., 2019), induction of apoptosis to inhibit tumor cell proliferation and angiogenesis (Rai, et al., 2015), regulation of endocrine and exocrine secretions including growth hormone in acromegaly, inhibition the secretion of insulin and glucagon (Murray, et al., 2015) and inhibition of the release of gastrointestinal hormones and thyroid-stimulating hormone (Theodoropoulou, et al., 2013).

The inhibitory effects of several hormones and growth factors as well as tumors growth factor are making somatostatin as a crucial tool for developing advanced treatments of patients with NETs. Commonly, the effect of somatostatin as an antitumor in NETs is based on their capability to exert antitumor activity through direct and indirect mechanisms (He, et al., 2019). The direct mechanisms include some biological processes in the cell such as induction pertussis toxin-sensitive, G-protein-coupled receptor (GPCR) intracellular signals, proapoptotic effect, and inhibition cell cycle (Chalabi, et al., 2014). One of the main processes in the indirect mechanisms of somatostatin is the inhibition of growth factor receptor signaling. In this process, protein tyrosine phosphatases (PTPs) has played an important role by dephosphorylating the growth factor in tyrosine kinase receptors (Theodoropoulou, et al., 2013).

On the other hand, somatostatin also exerts a number of indirect antitumor mechanisms such as inhibition of the secretion of hormones and growth factors, inhibition of the cell proliferation and angiogenesis by eliminating somatostatin receptor function, and modulation of the immune system to stimulate natural antitumor mechanism (Susini, et al., 2006; Chalabi, et al., 2014). However, somatostatin has a short half-life $\left(\mathrm{t}_{1 / 2}\right)$ in vivo (1-3 minutes), making it unsuitable for the treatment of NETs, hence some synthetic SSAs were developed including octreotide, lanreotide, vapreotide (Figure 2) (Marciniak, et al., 2017). To date, SSAs have 
been used to treat most NETs either for inhibiting the tumor growth or symptom relief (Cwikla, et al., 2015; Maqsood, et al., 2019).

Similar to native somatostatin, SSAs bind to SSTRs and induced a range of cellular effect, including antitumor activity (Sideris, et al., 2012). Octreotide was the first SSA approved and widely used for management of hormonal syndromes resulting from NETs. This analog has a strong affinity to SSTR-2 and SSTR-5 and showed antitumor properties in patients with NETs. Accumulating evidence showed octreotide long-acting repeatable (LAR) as well as lanreotide autogel (lanreotide ATG) have strong efficacy and well tolerated in NET patients (Godara, et al., 2019). In a clinical study by Rinke, et al., it was clearly demonstrated that treatment of NETs patients using octreotide LAR resulted in a median time to tumor progression of 14.3 months, higher than placebo groups (6 months). Additionally, stable disease was achieved in $66.7 \%$ of patients in the octreotide LAR group after 6 months of treatment. This number was higher than patients in placebo group (37.2\%) (Rinke, et al., 2009). Antiproliferative effects of lanreotide ATG has also been demonstrated in the efficacy and safety study in 30 patients with well-differentiated NETs. In this study, lanreotide ATG provided effective tumor stabilization in 24 patients $(89 \%)$ and median progression-free survival (PFS) of 12.9 months (Richard, 2013).

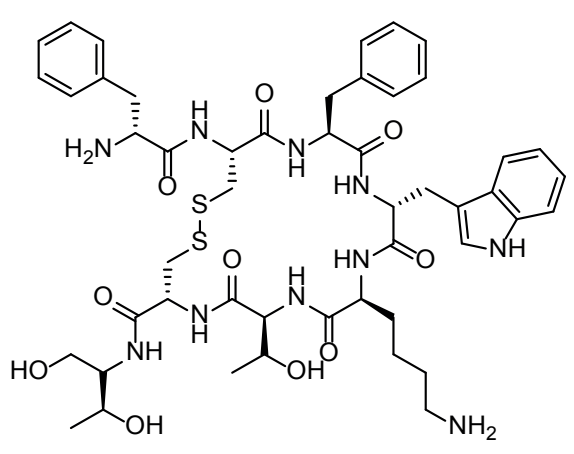

Octreotide

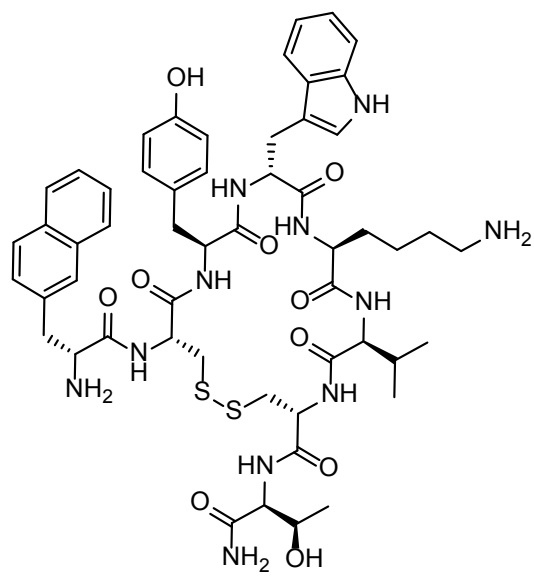

Lanreotide

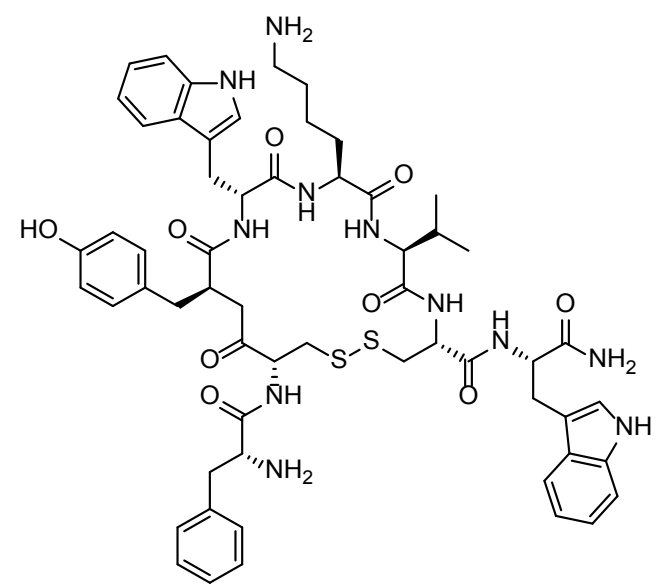

Figure 2. Chemical structure of several somatostatin SSAs (Gunther, et al., 2018).

Vapreotide 


\section{Peptide Receptor Radionuclide Therapy (PRRT)}

To date, some SSAs have also been exploited as PRRT for eradicating malignant cells in NETs. PRRT is a treatment that uses radioisotope attached to the SSA to deliver therapeutic dose of ionizing radiation to tumor cells (Dash, et al., 2015). The illustration of the PRRT mechanism to eradicate tumor cells is shown in Figure 3.

In the past ten years, some PRRT SSTRtargeting radionuclide therapies for clinical management of NETs were developed, including ${ }^{90} \mathrm{Y}-$ $\left[\right.$ DOTA $\left.^{0}, \quad \mathrm{Tyr}^{3}\right]$-octreotide $\quad\left({ }^{90} \mathrm{Y}\right.$-DOTA-TOC $)$, ${ }^{90} \mathrm{Y}-\left[\mathrm{DOTA}^{0}, \mathrm{Tyr}^{3}\right]$-octreotate $\left({ }^{90} \mathrm{Y}\right.$-DOTA-TATE), ${ }^{177} \mathrm{Lu}-\left[\mathrm{DOTA}^{0}, \quad \mathrm{Tyr}^{3}\right]$-octreotide $\quad{ }^{177} \mathrm{Lu}$-DOTATOC), ${ }^{177} \mathrm{Lu}-\left[\mathrm{DOTA}^{0}, \mathrm{Tyr}^{3}\right]$-octreotate $\quad\left({ }^{177} \mathrm{Lu}-\right.$ DOTA-TATE) (Gabriel, et al., 2007; Baum, et al., 2015). The idea of using ${ }^{90} \mathrm{Y}\left(\mathrm{t}_{1 / 2}=64.6\right.$ hours $)$ and ${ }^{177} \mathrm{Lu}\left(\mathrm{t}_{1 / 2}=160.8\right.$ hours $)$ as radionuclide therapy is based on their energy characteristics. ${ }^{177} \mathrm{Lu}$ emits $\beta-$ energy of $149 \mathrm{keV}$ and maximum particle range of $1.5 \mathrm{~mm}$, and therefore suitable for destroying small lesions of NETs. In contrast, ${ }^{90} \mathrm{Y}$ emits higher $\beta$ - energy of $2.28 \mathrm{MeV}$ and maximum particle range of $12 \mathrm{~mm}$ which more suitable for the eradication of bulky NETs. These characteristics make them well suited for PRRT of NETs (Perk, et al., 2005; Garkavij, et al., 2010; Fenwick, et al., 2014). However, the combination of ${ }^{90} \mathrm{Y}$ and ${ }^{177} \mathrm{Lu}$ may be the most effective for NETs treatment (Kwekkeboom, et al., 2006). Some alternative radionuclides (Table 1) can be considered for the treatment of NETs.

The growing number of studies indicates that various SSAs radiolabelled with ${ }^{90} \mathrm{Y}$ and ${ }^{177} \mathrm{Lu}$ have played an important role in NETs management strategy. For instance, ${ }^{90} \mathrm{Y}$-octreotide and ${ }^{177} \mathrm{Lu}-\mathrm{oc}-$ treotate demonstrated OR rates of 15-35\% (Baum, et al., 2015). Recent clinical studies indicated that PRRT can prolong the 5-year survival rate and improve quality of life factors in a considerable number of NETs patients. Moreover, PRRT achieved higher median 5-year survival compared to patients with untreated, conventional chemotherapy, transarterial chemoembolization (TACE) and transarterial embolization (TAE) (Bodei, et al., 2014). In particular, ${ }^{90} \mathrm{Y}$ has demonstrated significant contribution in the treatment of NETs as well as liver tumor without causing extreme dose to neighboring healthy tissues (Fenwick, et al., 2014). A study showed that therapy outcome of NETs using ${ }^{90} \mathrm{Y}-\mathrm{DOTA}-\mathrm{TOC}$ increased with adding of ${ }^{131} \mathrm{I}-\mathrm{MIBG}$ (Bushnell, et al., 2014).

In a more detailed study, it was shown that PRRT using ${ }^{90} \mathrm{Y}$-DOTA-TATE and ${ }^{90} \mathrm{Y}$-DOTATOC in patients with progressive metastatic NETs
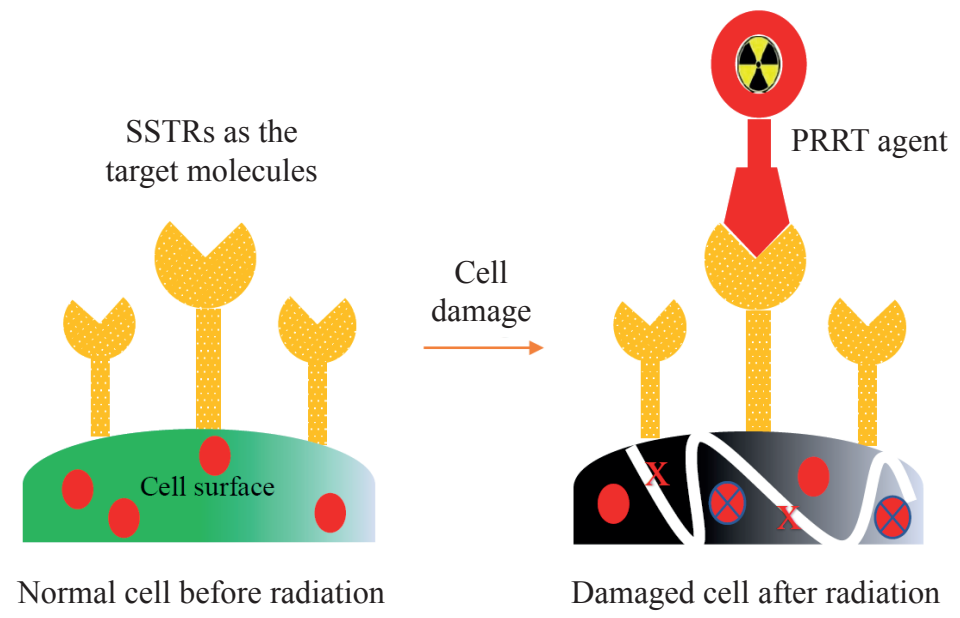

Figure 3. The schematic diagram illustrated the role of PRRT in the treatment of NETs. 
Indonesian Journal of Cancer Chemoprevention, June 2019

Table 1. Alternative radionuclides for PRRT.

\begin{tabular}{cccc}
\hline Radionuclide & Half-life $\left(\mathbf{T}_{\mathbf{1} / \mathbf{2}}\right)$ & Emissions & Reference \\
\hline${ }^{111} \mathrm{In}$ & 67.4 hours & $\gamma, \mathrm{e}^{-}$Auger & (Dash, et al., 2015$)$ \\
${ }^{67} \mathrm{Cu}$ & 61.9 hours & $\beta^{-}, \gamma$ & (Dash, et al., 2015$)$ \\
${ }^{47} \mathrm{Sc}$ & 82.1 hours & $\beta^{-}, \gamma$ & (Dash, et al., 2015$)$ \\
${ }^{161} \mathrm{~Tb}$ & 165.6 hours & $\beta^{-}, \gamma$ & (Dash, et al. , 2015) \\
${ }^{225} \mathrm{Ac}$ & 240.0 hours & $\alpha, \beta^{-}, \gamma$ & (Dash, et al. , 2015) \\
${ }^{213} \mathrm{Bi}$ & 0.77 hours & $\alpha, \beta^{-}$ & (Dash, et al. , 2015) \\
${ }^{64} \mathrm{Cu}$ & 12.8 hours & $\beta^{-}, \beta^{+}$ & (Cremonesi, et al. , 2006) \\
${ }^{166} \mathrm{Ho}$ & 26.8 hours & $\beta$ & (Cremonesi, et al. , 2006) \\
${ }^{188} \mathrm{Re}$ & 17.0 hours & $\beta^{-}$ & (Cremonesi, et al. , 2006) \\
\hline
\end{tabular}

were correlated with high values of positive response $(24.5 \%)$ and stable disease (47\%). Those values contributed to significant overall survival (OS) in patients. This study also revealed that renal toxicity was rare, however, the hematological toxicity was common (Vinjamuri, et al., 2013). Another clinical study showed that a single cycle of $5 \mathrm{GBq}{ }^{90} \mathrm{Y}$-DOTA-TATE was effective for treating metastatic NETs in the liver. Patient follow-up showed only single liver metastasis observed after 12 months of PRRT treatment (Baum, et al., 2012).

The current study also showed that ${ }^{177} \mathrm{Lu}-$ octreotate highly effective for treating advanced NETs of the small intestine and demonstrated stable disease in $47.5 \%$ patients and only $8.2 \%$ patients indicated with progressive disease (Sabet, et al., 2015). The positive contribution of ${ }^{177} \mathrm{Lu}-$ octreotate also reported by Ezziddin and co-workers with a median PFS and OS in advanced grade $1 / 2$ pNETs were 34 and 53 months, respectively. Furthermore, the partial response, minor response, stable disease and progressive disease were observed in 60.3, 11.8, 13.2 , and $14.7 \%$ patients, respectively (Ezziddin, et al., 2014). Kwekkeboom, et al., reported that a high value of stable disease of gastroenteropancreatic NETs (GEPNETs) was achieved by ${ }^{177} \mathrm{Lu}-\mathrm{DOTA}-$
TATE in 30 patients (40\%) (Kwekkeboom, et al., 2006). More recently, a study showed that longer OS was achieved in patients with bronchial NET or GEPNETs after PRRT with ${ }^{177} \mathrm{Lu}$-DOTA-TATE compared to control group (van der Zwan, et al., 2019).

The used of ${ }^{90} \mathrm{Y}$ and ${ }^{177} \mathrm{Lu}$ in combination formula showed more advantages than single treatment using ${ }^{90} \mathrm{Y}$ or ${ }^{177} \mathrm{Lu}$. In the pre-clinical study using rats tumor model, remarkable antitumor activity was achieved by the combination of ${ }^{90} \mathrm{Y}-\mathrm{SSA}$ and ${ }^{177} \mathrm{Lu}-\mathrm{SSA}(50 \%: 50 \%)$ compared to single treatment using ${ }^{90} \mathrm{Y}-\mathrm{SSA}$ or ${ }^{177} \mathrm{Lu}-\mathrm{SSA}$. This study also indicated that a combination of these radionuclides may become an effective strategy for eradication different size of tumor cells (de Jong, et al., 2005). Kunikowska and colleagues reported that the combination of ${ }^{90} \mathrm{Y}$ and ${ }^{177} \mathrm{Lu}$ demonstrated longer OS in NETs patients than single radionuclide treatment. In this study, 25 patients were administered with ${ }^{90} \mathrm{Y}$-DOTA-TATE (group A) and 25 patients were administered with the combination of ${ }^{90} \mathrm{Y} /{ }^{177} \mathrm{Lu}-$ DOTA-TATE $(1: 1)$ (group B). It was observed that the median event-free survival (EFS) in group A and B were 21.4 and 29.4 months, respectively. It also showed that $64 \%$ of patients from group 
B experienced stable disease after 12 treatments, whereas only $52 \%$ from group A (Kunikowska, et al., 2011). The comprehensive clinical study of PRRT in NETs treatment involving 27 patients has been reported by Matovic. This study used three different PRRTs: ${ }^{90} \mathrm{Y}-\mathrm{DOTA}-\mathrm{TOC},{ }^{177} \mathrm{Lu}-\mathrm{DOTA}-$ TATE, and the combination of ${ }^{90} \mathrm{Y}-\mathrm{DOTA}-\mathrm{TOC}$ and ${ }^{177} \mathrm{Lu}-\mathrm{DOTA} \neg \mathrm{TATE}$. It was concluded that the partial response, stable disease, and progressive disease were $25.9,63.0$, and $11.1 \%$, respectively (Matovic, 2012).

The main challenge for developing PRRT is the appearance of side effects due to the radiation exposures which can affect the normal functions of several organs and tissues including kidney, blood, and bone marrow. Villard, et al., reported that after a four cycles of therapy with ${ }^{90} \mathrm{Y}-\mathrm{DOTA}-\mathrm{TOC}, 15$ patients $(6.3 \%)$ experienced transient hematologic toxicities and 1 patient was diagnosed with acute myeloid leukemia. Interestingly, only 11 patients (4.4\%) experienced severe transient hematologic toxicities and negative myeloproliferative was observed after radiotherapy using the combination of ${ }^{90} \mathrm{Y}-\mathrm{DOTA}-\mathrm{TOC}$ and ${ }^{177} \mathrm{Lu}-\mathrm{DOTA}-\mathrm{TOC}$ (Villard, et al., 2012). To minimize the side effects, the role of excretion organs is crucial to accelerate the elimination of the radionuclide after the treatment. For example, the pathway involves reabsorption of the radionuclide by kidneys should be minimized. In order to overcome this issue, some organic compounds such as gelofucine and amino acids (e.g., lysine and arginine) have been used to reduce radionuclide uptake by the kidney (Vinjamuri, et al., 2013; Edgar, 2006).

\section{Combined Therapy in NETs}

The use of SSAs in routine clinical practice as an extensive treatment modality has revolutionized the management strategy of patients with NETs. In many patients, symptomatic relief and stabilization of tumor growth are achieved after the treatment using SSAs. However, partial or complete spontaneous tumor regression is considered a rare phenomenon, and therefore combined therapy using SSA featuring other therapy strategies are crucial to further enhance the clinical achievement in NETs patients (Uri, et al., 2018).

Currently, synergistic effects of combined therapy of SSA and PRRT (SSA-PRRT) have been reported. A retrospective observational study in 99 patients with G1/G2 NETs suggested that the median PFS for sequence therapy with SSA-chemotherapy was 20 months, while for SSA-PRRT was 30 months. Despite there was no statistical difference in PFS, those two sequence therapy provided higher PFS compared to SSA standard dose-SSA high dose (median PFS was not reached), but lower than SSA-everolimus (33 months). This study also showed that the number of side effects in patients was higher in SSA-everolimus and SSA-chemotherapy groups compared to SSA standard doseSSA high dose and SSA to PRRT groups (Faggiano, et al., 2019). Again, from a different retrospective observational study in 168 patients with GEPNETs, it was found that the median PFS for PRRT was 27 months in group I (PRRT monotherapy). Interestingly, the median PFS was increased to 48 months in group II when SSA was combined with PRRT (Yordanova, et al., 2018).

Furthermore, combined therapy using chemotherapy and PRRT (chemo-PRRT) has become an interesting therapeutic option for patients with advanced or relatively aggressive behaving metastatic NETs, especially when it is unlikely to achieve favorable response to a single treatment strategy (Basu, et al., 2016). The synergistic effect of combined PRRT using ${ }^{177} \mathrm{Lu}-\mathrm{DOTA} \neg$ TATE with chemotherapeutics agents capecitabine + temozolomide (CAPTEM) was reported in 30 patients with inoperable $\mathrm{G} 1 / \mathrm{G} 2$, progressive, functional or nonfunctional pNET. In this study, the median PFS was 48 months, while overall response rate (ORR) and disease control rate (DCR) were $80 \%$ and $100 \%$, respectively (Ramage, et al., 2018). These results suggest that combined therapy using chemo-PRRT is likely to increase the PFS in patients rather than single treatment with chemotherapy or PRRT alone. Moreover, in vivo study in mice bearing SSTR-ex- 
pressing H69 demonstrated that chemo-PRRT using TMZ and ${ }^{177} \mathrm{Lu}$-octreotate resulted in tumor size reduction.

Despite chemo-PRRT has shown encouraging results, negative interactions between the two treatments should be taken into consideration. For instance, chemotherapy agents might influence the SSTR-2 (PRRT target) expression on tumor cells. Additionally, tumor vasculature might be affected by chemotherapy whereas adequate tumor perfusion is required for local biodistribution and accumulation of PRRT agent (Bison, et al., 2015). Furthermore, in a study by Brieau, et al., a high occurrence of myelodysplastic syndrome (MDS) and acute myeloid leukemia (AML) in GEPNETs patients treated with PRRT after previous chemotherapy with alkylating agents were observed. This study suggests that the importance of previous chemotherapy before PRRT should be considered (Brieau, et al., 2016).

\section{CONCLUSION}

The management of NETs poses a serious challenge due to the heterogeneous clinical presentations and varying degree of aggressiveness. Therefore, the application of a combined treatment using two or more strategies may become a better option. More importantly, the treatment approach for NETs patients should be individualized based on each tumor characteristics. Over the last few years, some advanced managements strategies have been explored, including the treatment using SSAs. More recently, SSAs have also been used in PRRT strategy, especially for the treatment of inoperable or metastasized NETs. These strategies play an important role and have been proved as advanced techniques for treating NETs. Moreover, treatment using the combination of SSAs and PRRT or chemotherapy and PRRT showed encouraging outcome for the patients as compared with single treatment either using SSAs, chemotherapy or PRTT alone. As a result, NETs management based on SSAs has increased and likely to become a continuing trend in routine clinical practice. More in-depth study, however, is needed to further understanding the role of SSAs in the treatment of NETs.

\section{REFERENCES}

Adams, R.W., Dyson, P. and Barthelmes, L., 2014, Neuroendocrine Breast Tumours: Breast Cancer or Neuroendocrine Cancer Presenting in the Breast?, Breast., 23, 120-127.

Al-Chalabi, H., Cook, A., Ellis, C., Patel, C.N. and Scarsbrook, A. F., 2018, Feasibility of a Streamlined Imaging Protocol in Technetium-99m-Tektrotyd Somatostatin Receptor SPECT/CT, Clin Radiol., 73, 527-534.

Amr, B., Froghi, F., Edmond, M., Haq, K. and Thengungal Kochupapy, R., 2015, Management and Outcomes of Appendicular Neuroendocrine Tumours: Retrospective Review with 5-Year Follow-Up, Eur J Surg Oncol., 41, 1243-1246.

Baldelli, R., Barnabei, A., Rizza, L., Isidori, A. M., Rota, F., Di Giacinto, P., Paoloni, A., Torino, F., Corsello, S. M., Lenzi, A. and Appetecchia, M., 2014., Somatostatin Analogs Therapy in Gastroenteropancreatic Neuroendocrine Tumors: Current Aspects and New Perspectives, Front Endocrinol (Lausanne)., 5(7), 1-10.

Basu, S. and Ostwal, V., 2016, The Case for Combined Chemotherapy-Peptide Receptor Radionuclide Therapy (Chemo-PRRT) Strategy in Metastatic Neuroendocrine Tumor: Predicting and Looking at the Possible Case Scenarios, Eur J Nucl Med Mol Imaging, 43, 2453-2455.

Baum, R.P. and Kulkarni, H.R., 2012, Theranostics: From Molecular Imaging Using Ga-68 Labeled Tracers and PET/CT to Personalized Radionuclide Therapy - the Bad Berka Experience, Theranostics., 2(5), 437-447.

Baum, R.P., Puranik, A.D. and Kulkarini, H.R., 2015, Peptide Receptor Radionuclide Therapy (PRRT) of Neuroendocrine Tumors Current State and Future Perspectives, Int. J. Endo, Oncol., 2(2), 151-158.

Bison, S.M., Haeck, J.C., Bol, K., Koelewijn, S.J., Groen, H.C., Melis, M., Veenland, J.F., Bernsen, M.R. and De Jong, M., 2015, Optimization of Combined Temozolomide and Peptide Receptor 
Radionuclide Therapy (PRRT) in Mice after Multimodality Molecular Imaging Studies, EJNMMI Res., 5, 62.

Blaker, M., Schmitz, M., Gocht, A., Burghardt, S., Schulz, M., Broring, D.C., Pace, A., Greten, H. and De Weerth, A., 2004, Differential Expression of Somatostatin Receptor Subtypes in Hepatocellular Carcinomas, J Hepatol., 41, 112-118.

Bodei, L., Cremonesi, M., Kidd, M., Grana, C.M., Severi, S., Modlin, I.M. and Paganelli, G., 2014, Peptide Receptor Radionuclide Therapy for Advanced Neuroendocrine Tumors, Thorac Surg Clin., 24, 333-349.

Boora, G.K., Kanwar, R., Kulkarni, A.A., Pleticha, J., Ames, M., Schroth, G., Beutler, A.S. and Banck, M.S., 2015, Exome-Level Comparison of Primary Well-Differentiated Neuroendocrine Tumors and Their Cell Lines, Cancer Genet., 208, 374-381.

Boy, C., Heusner, T.A., Poeppel, T.D., Redmann-Bischofs, A., Unger, N., Jentzen, W., Brandau, W., Mann, K., Antoch, G., Bockisch, A. and Petersenn, S., 2011, ${ }^{68} \mathrm{Ga}$-DOTATOC PET/CT and Somatostatin Receptor (SST1-SST5) Expression in Normal Human Tissue: Correlation of SST2

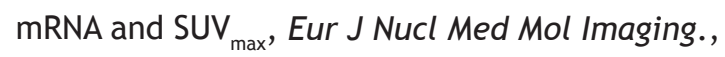
38, 1224-1236.

Brieau, B., Hentic, O., Lebtahi, R., Palazzo, M., Ben Reguiga, M., Rebours, V., Maire, F., Hammel, P., Ruszniewski, P. and Fenaux, P., 2016, High Risk of Myelodysplastic Syndrome and Acute Myeloid Leukemia after ${ }^{177} \mathrm{Lu}$-Octreotate PRRT in NET Patients Heavily Pretreated with Alkylating Chemotherapy, Endocr Relat Cancer., 23(5), L17-23.

Bushnell, D.L., Madsen, M.T., O'cdorisio, T., Menda, Y., Muzahir, S., Ryan, R. and O'dorisio, S., 2014, Feasibility and Advantage of Adding ${ }^{131}$ I-MIBG to ${ }^{90} Y$-DOTATOC for Treatment of Patients with Advanced Stage Neuroendocrine Tumors, EJNMMI Res., 4(38), 1-8.

Cakir, M., Dworakowska, D. and Grossman, A., 2010, Somatostatin Receptor Biology in Neuroendocrine and Pituitary Tumours: Part 2--Clinical Implications, J Cell Mol Med., 14(11), 25852591.

Chalabi, M., Duluc, C., Caron, P., Vezzosi, D., Guillermet-Guibert, J., Pyronnet, S. and Bousquet, C.,
2014, Somatostatin Analogs: Does Pharmacology Impact Antitumor Efficacy?, Trends Endocrinol Metab., 25(3), 115-127.

Chinezu, L., Vasiljevic, A., Jouanneau, E., Francois, P., Borda, A., Trouillas, J. and Raverot, G. 2014. Expression of Somatostatin Receptors, SSTR2a and SSTR5, in 108 Endocrine Pituitary Tumors Using Immunohistochemical Detection with New Specific Monoclonal Antibodies, Hum Pathol., 45, 71-77.

Cremonesi, M., Ferrari, M., Bodei, L., Tosi, G. and Paganelli, G., 2006, Dosimetry in Peptide Radionuclide Receptor Therapy: A Review, J Nucl Med., 47(9), 1467-1475.

Cwikla, J.B., Bodei, L., Kolasinska-Cwikla, A., Sankowski, A., Modlin, I.M. and Kidd, M., 2015, Circulating Transcript Analysis (NETEST) in GEPNETS Treated with Somatostatin Analogs Defines Therapy, J Clin Endocrinol Metab., 100(11), E1437-45.

Dash, A., Chakraborty, S., Pillai, M.R. and Knapp, F.F., 2015, Peptide Receptor Radionuclide Therapy: An Overview, Cancer Biother Radiopharm, 30(2), 47-71.

De Jong, M., Breeman, W.A.P., Valkema, R., Bernard, B.F. and Krenning, E.P., 2005, Combination Radionuclide Therapy Using ${ }^{177} \mathrm{Lu}$ - and ${ }^{90} \mathrm{Y}$-Labeled Somatostatin Analogs, J Nucl Med., 46(1), 13S-17S.

Dilz, L.M., Denecke, T., Steffen, I.G., Prasad, V., Von Weikersthal, L.F., Pape, U.F., Wiedenmann, B. and Pavel, M., 2015, Streptozocin/5-Fluorouracil Chemotherapy Is Associated with Durable Response in Patients with Advanced Pancreatic Neuroendocrine Tumours, Eur J Cancer., 51, 1253-1262.

Edgar, R., De Jong, M., Valkema, R., Kwekkeboom, D., 2006, Inhibition of Kidney Uptake of Radiolabeled Somatostatin Analogs: Amino Acid of Gelofusine?, J Nucl Med., 47(10), 1730-1731.

Ezziddin, S., Khalaf, F., Vanezi, M., Haslerud, T., Mayer, K., Al Zreiqat, A., Willinek, W., Biersack, H.J. and Sabet, A., 2014, Outcome of Peptide Receptor Radionuclide Therapy with 177Lu-Octreotate in Advanced Grade 1/2 Pancreatic Neuroendocrine Tumours, Eur J Nucl Med Mol Imaging., 41, 925-933. 
Indonesian Journal of Cancer Chemoprevention, June 2019 ISSN: 2088-0197

e-ISSN: $2355-8989$

Faggiano, A., Di Maio, S., Mocerino, C., Ottaviano, M., De Divitiis, C., Guarnotta, V., Dolce, P., Modica, R., Puliafito, I., Tozzi, L., Di Sarno, A., Leo, S., Riccardi, F., Palmieri, G., Tafuto, S., Bianco, A., Badalamenti, G., Colao, A. and Elios., 2019, Therapeutic Sequences in Patients with Grade 1-2 Neuroendocrine Tumors (NET): An Observational Multicenter Study from the Elios Group, Endocrine., 1-8.

Fani, M., Nicolas, G. P. and Wild, D., 2017, Somatostatin Receptor Antagonists for Imaging and Therapy, J Nucl Med., 58(9), 61S-66S.

Fenwick, A., Baker, M., Ferreira, K. and Keightley, J., 2014, Comparison of ${ }^{90} \mathrm{Y}$ and ${ }^{177} \mathrm{Lu}$ Measurement Capability in Uk and European Hospitals, Appl Radiat Isot., 87, 10-13.

Frilling, A., Smith, G., Clift, A.K. and Martin, J., 2014, Capsule Endoscopy to Detect Primary Tumour Site in Metastatic Neuroendocrine Tumours, Dig Liver Dis., 46, 1038-1042.

Gabriel, M., Decristoforo, C., Kendler, D., Dobrozemsky, G., Heute, D., Uprimny, C., Kovacs, P., Von Guggenberg, E., Bale, R. and Virgolini, I.J., 2007, ${ }^{68} \mathrm{Ga}$-DOTA-Tyr ${ }^{3}$-Octreotide PET in Neuroendocrine Tumors: Comparison with Somatostatin Receptor Scintigraphy and CT, J Nucl Med., 48(4), 508-518.

Gandomkar, M., Najafi, R., Shafiei, M., Mazidi, M. and Ebrahimi, S.E., 2007, Preclinical Evaluation of [99m Tc/Edda/Tricine/Hynic $\left.{ }^{0}, 1-\mathrm{Nal}^{3}, \mathrm{Thr}^{8}\right]$-Octreotide as a New Analogue in the Detection of Somatostatin-Receptor-Positive Tumors, Nucl Med Biol., 34, 651-657.

García-Talavera, P., Ruano, R., Rioja, M.E., Cordero, J.M., Razola, P. and Vidal-Sicart, S., 2014, Radioguided Surgery in Neuroendocrine Tumors. A Review of the Literature, Rev Esp Med Nucl Ima (English Edition)., 33(6), 358-365.

Garkavij, M., Nickel, M., Sjogreen-Gleisner, K., Ljungberg, M., Ohlsson, T., Wingardh, K., Strand, S.

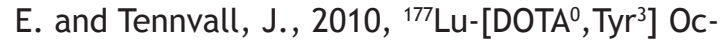
treotate Therapy in Patients with Disseminated Neuroendocrine Tumors: Analysis of Dosimetry with Impact on Future Therapeutic Strategy, Cancer., 116, 1084-1092.

Godara, A., Siddiqui, N.S., Byrne, M.M. and Saif, M.W., 2019, The Safety of Lanreotide for Neu- roendocrine Tumor, Expert Opin Drug Saf., 18(1), 1-10.

Gould, E.N., Johnson, L.R., Traslavina, R.P. and Mohr, F.C., 2013, Neuroendocrine Tumour at the Carina of a Dog, J Comp Pathol., 149, 233-236.

Ha, J. and Tan, W.A., 2012, Gastrointestinal Carcinoid Tumours: A Review, Journal of Gastrointestinal \& Digestive System, 2(2), 1-7.

He, R., Finan. B., Mayer, J.P. and DiMarchi, D., 2019, Peptide Conjugates with Small Molecules Designed to Enhance Efficacy and Safety, Molecules., 24, 1-34.

Jayasena, C.N. and Dhillo, W.S., 2013, Carcinoid Syndrome and Neuroendocrine Tumours, Medicine., 41(10), 566-569.

Kaltsas, G.A., Besser, G.M. and Grossman, A.B., 2004, The Diagnosis and Medical Management of Advanced Neuroendocrine Tumors, Endocr Rev., 25(3), 458-511.

Kayani, I., Bomanji, J.B., Groves, A., Conway, G., Gacinovic, S., Win, T., Dickson, J., Caplin, M. and Ell, P.J., 2008, Functional Imaging of Neuroendocrine Tumors with Combined PET/CT Using ${ }^{68} \mathrm{Ga}$-DOTATATE (DOTA-Dphe1, Tyr ${ }^{3}$-Octreotate) and ${ }^{18} \mathrm{~F}-\mathrm{FDG}$, Cancer, 112(11), 2447-2455.

Khan, M.U. and Coleman, R.E., 2008, Diagnosis and Therapy of Carcinoid Tumors-Current State of the Art and Future Directions, Nucl Med Biol., 35, S77-S91.

Kidd, M., Modlin, I.M., Bodei, L. and Drozdov, I., 2015, Decoding the Molecular and Mutational Ambiguities of Gastroenteropancreatic Neuroendocrine Neoplasm Pathobiology, Cell Mol Gastroenterol Hepatol., 1(2), 131-153.

Korse, C.M., Taal, B.G., Van Velthuysen, M.L. and Visser, O., 2013, Incidence and Survival of Neuroendocrine Tumours in the Netherlands According to Histological Grade: Experience of Two Decades of Cancer Registry, Eur J Cancer., 49, 1975-1983.

Kunikowska, J., Krolicki, L., Hubalewska-Dydejczyk, A., Mikolajczak, R., Sowa-Staszczak, A. and Pawlak, D., 2011, Clinical Results of Radionuclide Therapy of Neuroendocrine Tumours with ${ }^{90} \mathrm{Y}$-DOTATATE and Tandem ${ }^{90} \mathrm{Y} /{ }^{177} \mathrm{Lu}$-DOTATATE: Which Is a Better Therapy Option?, Eur J Nucl Med Mol Imaging., 38, 1788-1797. 
Kwekkeboom, D., Mueller-Brand, J., Paganelli, G., Anthony, L.B., Pauwels, S., Kvols, L.K., O'dorisio, T.M., Valkema, R., Bodei, L., Chinol, M., Maecke, H.R. and Krenning, E.P., 2006, Overview of Results of Peptide Receptor Radionuclide Therapy with 3 Radiolabeled Somatostatin Analogs, J Nucl Med., 46(1), 62S-66S.

Luke, C., Price, T., Townsend, A., Karapetis, C., Kotasek, D., Singhal, N., Tracey, E. and Roder, D., 2010, Epidemiology of Neuroendocrine Cancers in an Australian Population, Cancer Causes Control., 21, 931-938.

Maqsood, M.H., Din, A.T. and Khan, A.H., 2019, Neuroendocrine Tumor Therapy with Lutetium-177: A Literature Review, Cureus., 11(1), 1-7.

Marciniak, A. and Brasun, J., 2017, Somatostatin Analogues Labeled with Copper Radioisotopes: Current Status, J Radioanal Nucl Chem., 313, 279-289.

Matovic, M., 2012, Peptide Receptor Radionuclide Therapy of Neuroendocrine Tumors: Case Series, Archive of Oncology., 20(3-4), 143-148.

Mayo, S.C., De Jong, M.C., Pulitano, C., Clary, B. M., Reddy, S.K., Gamblin, T.C., Celinksi, S.A., Kooby, D.A., Staley, C.A., Stokes, J.B., Chu, C.K., Ferrero, A., Schulick, R.D., Choti, M.A., Mentha, G., Strub, J., Bauer, T.W., Adams, R.B., Aldrighetti, L., Capussotti, L. and Pawlik, T.M., 2010, Surgical Management of Hepatic Neuroendocrine Tumor Metastasis: Results from an International Multi-Institutional Analysis, Ann Surg Oncol., 17, 3129-3136.

Modlin, I.M., Lye, K.D. and Kidd, M., 2003, A 5-Decade Analysis of 13,715 Carcinoid Tumors, Cancer., 97(4), 934-959.

Moertel, C.G., 1983, Treatment of the Carcinoid Tumor and the Malignant Carcinoid Syndrome, J Clin Oncol., 1(11), 727-740.

Murray, P.G., Higham, C.E. and Clayton, P.E., 2015, 60 Years of Neuroendocrinology: The Hypothalamo-Gh Axis: The Past 60 Years, J Endocrinol., 226(2), T123-40.

Nave, H., Mössinger, E., Feist, H., Lang, H. and Raab, H.R., 2001, Surgery as Primary Treatment in Patients with Liver Metastases from Carcinoid Tumors: A Retrospective, Unicentric Study over 13 Years, Surgery, 129(2), 170-175.
Oberg, K.E., 2012, The Management of Neuroendocrine Tumours: Current and Future Medical Therapy Options, Clin Oncol (R Coll Radiol)., 24, 282-293.

Owonikoko, T.K., Zhang, G., Lallani, S.B., Chen, Z., Martinson, D.E., Khuri, F.R., Lonial, S., Marcus, A. and Sun, S.Y., 2019, Evaluation of Preclinical Efficacy of Everolimus and Pasireotide in Thyroid Cancer Cell Lines and Xenograft Models, Plos One., 1-15.

Patel, Y.C., Greenwood, M.T., Panetta, R., Demchyshyn, L., Niznik, H. and Srikant, C.B., 1995, The Somatostatin Receptor Family, Life Sci., 57(13), 1249-1265.

Pathak, S., Dash, I., Taylor, M.R. and Poston, G.J., 2013, The Surgical Management of Neuroendocrine Tumour Hepatic Metastases, Eur J Surg Oncol., 39, 224-228.

Peixoto, R.D., Noonan, K.L., Pavlovich, P., Kennecke, H.F. and Lim, H.J., 2014, Outcomes of Patients Treated with Capecitabine and Temozolamide for Advanced Pancreatic Neuroendocrine Tumors (pNETs) and Non-pNETs, J Gastrointest Oncol., 5(4), 247-252.

Perk, L., Gerard, W.M., Vosjan, M., Walsum, M.S., Tijink, B., Leemans, R. and Dongen, G., 2005, ${ }^{89} \mathrm{Zr}$ as a PET Surrogate Radioisotope for Scouting Biodistribution of the Therapeutic Radiometals ${ }^{90} \mathrm{Y}$ and ${ }^{177} \mathrm{Lu}$ in Tumor-Bearing Nude Mice after Coupling to the Internalizing Antibody Cetuximab, J Nucl Med., 46(11), 1898-1906.

Rai, U., Thrimawithana, T. R., Valery, C. and Young, S.A, 2015, Therapeutic Uses of Somatostatin and Its Analogues: Current View and Potential Applications, Pharmacol Ther., 152, 98-110.

Ramage, J., Naraev, B.G. and Halfdanarson, T.R., 2018, Peptide Receptor Radionuclide Therapy for Patients with Advanced Pancreatic Neuroendocrine Tumors, Semin Oncol., 45, 236-248.

Richard, M.M., Massuti, B., Pineda, E., Alonso, V., Marmol, M., Castellano, D., Fonseca, E., Galan, A., Llanos, M., Sala, M.A., Pericay, C., Rivera, F., Sastre, J., Segura, A., Quindos, M. and Maisonoble, P., 2013, Antiproliferative Effects of Lanreotide Autogel in Patients with Progressive, Well-Differentiated Neuroendocrine Tumours: A Spanish, Kulticentre, Open-Label, Single Arm 
Indonesian Journal of Cancer Chemoprevention, June 2019 ISSN: 2088-0197

e-ISSN: $2355-8989$

Phase II Study, BMC Cancer, 13, 1-9.

Rinke, A., Muller, H.H., Schade-Brittinger, C., Klose, K.J., Barth, P., Wied, M., Mayer, C., Aminossadati, B., Pape, U.F., Blaker, M., Harder, J., Arnold, C., Gress, T., Arnold, R. and Group, P. S., 2009, Placebo-Controlled, Double-Blind, Prospective, Randomized Study on the Effect of Octreotide Lar in the Control of Tumor Growth in Patients with Metastatic Neuroendocrine Midgut Tumors: A Report from the Promid Study Group, J Clin Oncol., 27(28), 4656-4663.

Sabet, A., Dautzenberg, K., Haslerud, T., Aouf, A., Sabet, A., Simon, B., Mayer, K., Biersack, H.J. and Ezziddin, S., 2015, Specific Efficacy of Peptide Receptor Radionuclide Therapy with ${ }^{177}$ Lu-Octreotate in Advanced Neuroendocrine Tumours of the Small Intestine, Eur J Nucl Med Mol Imaging., 42, 1238-1246.

Sideris, L., Dube, P. and Rinke, A., 2012, Antitumor Effects of Somatostatin Analogs in Neuroendocrine Tumors, Oncologist., 17, 747-755.

Skuladottir, H., Hirsch, F.R., Hansen, H.H. and Olsen, J.H., 2002, Pulmonary Neuroendocrine Tumors Incidence and Prognosis of Histological Subtypes. A Population-Based Study in Denmark, Lung Cancer, 37, 127-135.

Susini, C. and Buscail, L., 2006, Rationale for the Use of Somatostatin Analogs as Antitumor Agents, Ann Oncol., 17(12), 1733-1742.

Theodoropoulou, M. and Stalla, G.K., 2013, Somatostatin Receptors: From Signaling to Clinical Practice, Front Neuroendocrinol, 34, 228-252.

Uri, I. and Grozinsky-Glasberg, S., 2018, Current Treatment Strategies for Patients with Advanced Gastroenteropancreatic Neuroendocrine Tumors (GEP-NETs), Clin Diabetes Endocrinol, 4, 16.

Van der Zwan, J.M., Trama, A., Otter, R., Larranaga, N., Tavilla, A., Marcos-Gragera, R., Dei Tos, A.P., Baudin, E., Poston, G., Links, T. and Rarecare, W.G., 2013, Rare Neuroendocrine Tumours: Results of the Surveillance of Rare Cancers in Europe Project, Eur J Cancer, 49, 2565-2578.

Van der Zwan, W.A., Brabander, T., Kam, B.L.R., Teunissen, J.J.M., Feelders, R.A., Hofland, J., Krenning, E.P., de Herder, W.W., 2019, Salvage
Peptide Receptor Radionuclide Therapy with [ ${ }^{177}$ Lu-DOTA, Tyr $\left.{ }^{3}\right]$ Octreotate in Patients with Bronchial and Gastroenteropancreatic Neuroendocrine Tumours, Eur J Nucl Med Mol Imaging., 46, 704-717.

Villard, L., Romer, A., Marincek, N., Brunner, P., Koller, M.T., Schindler, C., Ng, Q.K., Macke, H.R., Muller-Brand, J., Rochlitz, C., Briel, M. and Walter, M.A., 2012, Cohort Study of Somatostatin-Based Radiopeptide Therapy with $\left[\left({ }^{90}\right)\right.$ Y-DOTA]-TOC Versus $\left[\left({ }^{90}\right)\right.$ Y-DOTA]-TOC Plus $\left[\left({ }^{177}\right)\right.$ Lu-DOTA]-TOC in Neuroendocrine Cancers, J Clin Oncol., 30(10), 1100-1106.

Vinjamuri, S., Gilbert, T.M., Banks, M., Mckane, G., Maltby, P., Poston, G., Weissman, H., Palmer, D.H., Vora, J., Pritchard, D.M. and Cuthbertson, D.J., 2013, Peptide Receptor Radionuclide Therapy with $\left({ }^{90}\right)$ Y-DOTATATE $/\left({ }^{90}\right)$ Y-DOTATOC in Patients with Progressive Metastatic Neuroendocrine Tumours: Assessment of Response, Survival and Toxicity, Br J Cancer, 108, 1440-1448.

Walter, T., Brixi-Benmansour, H., Lombard-Bohas, C. and Cadiot, G., 2012, New Treatment Strategies in Advanced Neuroendocrine Tumours, Dig Liver Dis. , 44, 95-105.

Wang, X., Fani, M., Schulz, S., Rivier, J., Reubi, J.C. and Maecke, H.R., 2012, Comprehensive Evaluation of a Somatostatin-Based Radiolabelled Antagonist for Diagnostic Imaging and Radionuclide Therapy, Eur J Nucl Med Mol Imaging, 39, 1876-1885.

Yao, J.C., Hassan, M., Phan, A., Dagohoy, C., Leary, C., Mares, J.E., Abdalla, E.K., Fleming, J.B., Vauthey, J.N., Rashid, A. and Evans, D.B., 2008, One Hundred Years after "Carcinoid": Epidemiology of and Prognostic Factors for Neuroendocrine Tumors in 35,825 Cases in the United States, J Clin Oncol., 26(18), 3063-3072.

Yordanova, A., Wicharz, M.M., Mayer, K., Brossart, P., Gonzalez-Carmona, M.A., Strassburg, C.P., Fimmers, R., Essler, M. and Ahmadzadehfar, H., 2018, The Role of Adding Somatostatin Analogues to Peptide Receptor Radionuclide Therapy as a Combination and Maintenance Therapy, Clin Cancer Res., 24, 4672-4679. 